\title{
Cotidiano de mulheres colostomizadas e o impacto na sexualidade
}

Daily life of colostomized women and the impact on sexuality

La vida cotidiana de las mujeres colostomizadas y el impacto em la sexualidade

\author{
Nathália da Silva Cruz ${ }^{1 *}$, Lucia de Medeiros Taveira²
}

Como citar esse artigo. Cruz, NS; Taveira, LM. Cotidiano de mulheres colostomizadas e o impacto na sexualidade. Revista Pró-UniverSUS. 2020 Jul./Dez.; 11 (2): 121-128.

\begin{abstract}
Resumo
Objetivo: Conhecer a interferência no cotidiano e na expressão da sexualidade de mulheres com colostomias. Metodologia: Trata-se de uma revisão descritiva, exploratória e qualitativa. Os dados foram coletados na base de dados BVS, Scielo e LILACS. Os critérios de inclusão para a realização do trabalho foram artigos brasileiros e estrangeiros publicados entre os anos de 2015 e 2019. Dessa forma, nove artigos foram selecionados para interpretação, análise, discussão e estruturação deste trabalho. Resultados: Parte significante das mudanças concentra-se no controle fecal e de gases, tal como problemas na prática de atividades do dia a dia, no exercício profissional e em momentos de lazer. Quanto à sexualidade, a mulher colostomizada pode apresentar barreiras construídas por ela mesma, como os sentimentos de insegurança e vergonha do próprio corpo, resultantes do abalo psicológico ocasionado pela mudança da imagem corporal. Conclusão: É muito importante que sejam implementadas estratégias educativas para mulheres estomizadas e seus parceiros, a fim de que sejam auxiliados a superarem suas dificuldades, com o intuito de se tornarem capazes de vivenciar sua sexualidade e suas vidas de forma agradável. A equipe de enfermagem exerce papel fundamental no acompanhamento e assistência à essas pacientes, proporcionando o estímulo do autocuidado e fortalecimento da autoestima e reintegração social.
\end{abstract}

Palavras-chave: Estomia; Colostomia; Sexualidade; Saúde da Mulher; Imagem Corporal.

\begin{abstract}
Objective: To know the interference in the daily life and expression of sexuality in women with colostomies. Methodology: This is a descriptive, exploratory and qualitative review. Data were collected from the VHL, Scielo and LILACS database. The inclusion criteria for the study were Brazilian and foreign articles published between 2015 and 2019. Thus, 9 articles were selected for interpretation, analysis, discussion and structuring of this work. Outcomes: A significant part of the changes focus on fecal and gas control, such as problems with day-to-day activities, professional practice and leisure time. As for sexuality, the colostomized woman may present barriers constructed by herself, such os feelings of insecurity and shame of her own body, resulting from the psychological shaking caused by the change in body image. Conclusion: It is very important that educational strategies are implemented for ostomized women and their partners, so that they are helped to overcome their difficulties in order to be able to experience their sexuality and their lives in a pleasant way. The nursing team plays a fundamental role in monitoring and assisting these patients, providing the encouragement of self-care and strengthening self-esteem and social reintegration.

Keywords: Ostomy; Colostomy; Sexuality; Women’s Health; Bodyimage.
\end{abstract}

\section{Resumen}

Objetivo: Conocer la interferencia en la vida cotidiana y en la expresión de la sexualidad de las mujeres con colostomías. Metodología: Esta es una revisión descriptiva, exploratoria y cualitativa. Los datos fueron recolectados en las bases de datos VHL, SciELO y LILACS. Los criterios de inclusión para llevar a cabo el trabajo fueron los artículos brasileños y extranjeros publicados entre los años 2015 y 2019. Por lo tanto, se seleccionaron 9 artículos para la interpretación, análisis, discusión y estructuración de este trabajo. Resultados: Una parte importante de los cambios se concentra en el control de gases y heces, así como en los problemas en la práctica de las actividades diarias, en la práctica profesional y en los momentos de ocio. En cuanto a la sexualidad, la mujer colostomizada puede presentar barreras construidas por ella misma, como sentimientos de inseguridad y vergüenza en su propio cuerpo, como resultado del shock psicológico causado por el cambio en la imagen corporal. Conclusión: Es muy importante que se implementen estrategias educativas para las mujeres ostomizadas. sus parejas, para ser ayudadas a superar sus dificultades, para poder experimentar su sexualidad y sus vidas de una manera agradable. El equipo de enfermería desempeña un papel fundamental en el monitoreo y asistencia a estos pacientes, proporcionando el estímulo autocuidado y fortalecimiento de la autoestima y reintegración social.

Palabras clave: Ostomía; Colostomía Sexualidad; La salud de la mujer; Imagen Corporal.

Afiliação dos autores:

${ }^{1}$ Enfermeira pela Universidade Paulista, Brasília-DF, Brasil. ORCID: https://orcid.org/0000-0003-4941-6560

${ }^{2}$ Professora Titular do Curso de Enfermagem da Universidade Paulista, Campus Brasília-DF, Brasil. https://orcid.org/0000-0001-9907-2183

* Email de correspondencia: nathalya_cruz@hotmail.com 


\section{Introdução}

A estomia é uma intervenção cirúrgica realizada para exteriorizar um órgão oco, estabelecendo uma comunicação com o meio externo. A principal finalidade em estomias intestinais é o desvio da eliminação de fezes e gases, onde normalmente ocorre a exteriorização do cólon (colostomia) ou do íleo (ileostomia). ${ }^{1}$ Tratase de uma cirurgia agressiva capaz de acarretar várias mudanças no aspecto físico e psicossocial, fisiologia corporal e no estilo de vida da pessoa. ${ }^{2}$

A construção de uma estomia intestinal pode ser provocada por diversas doenças que afetam órgãos do sistema digestório como as neoplasias, diverticulites, doenças inflamatórias, além de causas externas em que podem ocasionar lesões intestinais, seja por ferimento de arma de fogo ou arma branca ou traumatismo decorrente a violência interpessoal ou acidentes automobilísticos. ${ }^{1}$

O processo cirúrgico para a construção do estoma é encarado de forma traumática, pois acarreta alterações dolorosas no estilo de vida dos estomizados. ${ }^{3}$ Além das eliminações serem modificadas, ocorrem mudanças no cotidiano, hábitos alimentares, renovação de crenças e valores, resultando alteração na qualidade de vida desses sujeitos, principalmente nos aspectos físicos e sociais. ${ }^{4,5} \mathrm{O}$ surgimento da estomia implica grandes transformações pessoais, podendo surgir estresse quando exigências aparecem sobrecarregando ou ultrapassando capacidades adaptativas de um sujeito. ${ }^{6}$

Diante da complexidade dessa situação, um trabalho interdisciplinar que atua por meio de uma escuta ativa e qualificada dos profissionais, é fundamental e facilita no processo de reabilitação dos estomizados. ${ }^{7}$

O portador de estomia intestinal carece de cuidados de enfermagem diferenciados e especializados, considerando todos os impactos que essa condição implica em suas vidas: $35 \%$ deixaram de trabalhar, $98 \%$ relataram problemas na vida sexual. ${ }^{8}$

Tratando-se da assistência de enfermagem, a consulta em estomaterapia tem como objetivo o acompanhamento do estomizado desde o pré-operatório até sua autonomia, reduzindo e resolvendo problemas implícitos, amparando-o e à família na recuperação e obtenção da melhor qualidade de vida, pois, devido às novas situações vivenciadas pelo estomizado, o sofrimento enfrentado pelo mesmo acarreta um potencial desajustamento, e o enfermeiro é um profissional que atua positivamente nesse processo de transição, que necessita adaptação e aceitação à nova condição de vida. ${ }^{9}$

Diante disso, dificuldades de aceitação ocorrem frente à alteração da forma e da percepção de corpo, com diferentes níveis de intensidade e repercussões, até mesmo nos aspectos relacionados aos cuidados com a saúde sexual. ${ }^{10}$ Após a cirurgia, muitas pessoas apresentam-se abaladas física e emocionalmente, reconhecendo-se menos atraentes e sensuais, situação que afeta negativamente na vivência da sexualidade. ${ }^{11}$

Percorrendo diversas fases do viver humano, a sexualidade transcende a necessidade fisiológica e tem relação direta com a manifestação do desejo. Referese à emoção que o sexo pode provocar, ultrapassando definições físicas. Mas também envolve quesitos físicos como a disfunção erétil, perda da libido, dor, entre outros, tornando a sexualidade e $\mathrm{o}$ ato sexual ocasionais em sua vida. ${ }^{12}$

A sexualidade faz parte da personalidade de cada pessoa, é uma necessidade vital do ser humano que não pode ser isolada dos outros aspectos da vida. Manifestase como necessidade fisiológica onde o desejo e a atração têm relação direta. $\mathrm{O}$ sexo provoca emoções e ultrapassa concepções físicas que intermedeia a vida do ser, tendo inúmeras faces, e que abrange significativa carga de subjetividade. ${ }^{13}$

Portanto, a questão que conduziu esse estudo foi: Quais os impactos sofridos no cotidiano e na vida sexual de mulheres colostomizadas? A partir desta, objetivou-se conhecer por meio da literatura científica o cotidiano e a interferência na sexualidade de mulheres colostomizadas. Acredita-se que é relevante realizar este trabalho, pois ressalta a vivência dessas mulheres com sua situação de saúde, salientando ainda que, as mulheres de modo geral, se preocupam muito com a imagem corporal, e ao buscar conhecer como elas lidam com a adaptação da colostomia, poderemos também compreender todo o decorrer do processo de aceitação e adaptação.

\section{Materiais e Métodos}

Trata-se de uma revisão integrativa da literatura, de caráter descritivo exploratório e qualitativo, com o intuito de conhecer como mulheres colostomizadas vivenciam o dia a dia e como lidam com os impactos sofridos na sexualidade. Para a pesquisa, utilizaramse as bases de dados Biblioteca Virtual em Saúde (BVS), Scientific Eletronic Library Online (SCIELO) e Latino Americana e do Caribe em Ciências da Saúde (LILACS).

Os critérios de inclusão foram artigos em português e inglês no período de janeiro de 2015 a 2019 , dentro da temática do estudo. Foram excluídos artigos duplicados, publicados há mais de cinco anos e que cuja metodologia e temática não eram proporcionais à proposta deste estudo.

\section{Resultados e discussões}

\section{Descrição geral dos artigos selecionados}

Após a leitura minuciosa de todos os artigos selecionados, deu-se a realização dos resultados e discussões descritos a seguir na Tabela 1. 
Tabela 1. Distribuição dos artigos de acordo com o título, autor (es), objetivos, métodos, conclusão e ano de conclusão.

\begin{tabular}{|c|c|c|c|c|c|c|}
\hline & TÍTULO & AUTOR(ES) & OBJETIVO & MÉTODO & CONCLUSÃO & ANO \\
\hline Artigo 1 & $\begin{array}{l}\text { Vida e sexualidade } \\
\text { de mulheres estomi- } \\
\text { zadas: subsídios à } \\
\text { enfermagem. }\end{array}$ & $\begin{array}{l}\text { Marina S; Camila } \\
\text { D; Giovana C. }\end{array}$ & $\begin{array}{l}\text { O estudo teve por ob- } \\
\text { jetivo conhecer como } \\
\text { a estomização inter- } \\
\text { fere na expressão da } \\
\text { sexualidade de mu- } \\
\text { lheres estomizadas. }\end{array}$ & $\begin{array}{l}\text { Pesquisa qualita- } \\
\text { tiva. }\end{array}$ & $\begin{array}{l}\text { É importante que os } \\
\text { profissionais da saú- } \\
\text { de implementem es- } \\
\text { tratégias educativas } \\
\text { para mulheres esto- } \\
\text { mizadas e seus com- } \\
\text { panheiros. }\end{array}$ & 2016 \\
\hline
\end{tabular}

\begin{tabular}{|c|c|c|c|c|c|}
\hline Artigo 2 & $\begin{array}{l}\text { Qualidade de vida } \\
\text { da pessoa estomiza- } \\
\text { da: relação com os } \\
\text { cuidados prestados } \\
\text { na consulta de enfer- } \\
\text { magem de estoma- }\end{array}$ & $\begin{array}{l}\text { Liliana S; Amân- } \\
\text { cio A; Elisabete P. }\end{array}$ & $\begin{array}{l}\text { Analisar a relação } \\
\text { entre a Qualidade de } \\
\text { Vida (QV) e os cuida- } \\
\text { dos prestados na con- } \\
\text { sulta de enfermagem } \\
\text { de estomaterapia. }\end{array}$ & $\begin{array}{l}\text { Estudo descritivo- } \\
\text { correlacional. }\end{array}$ & $\begin{array}{l}\text { Existe relação esta- } \\
\text { tística entre QV, o } \\
\text { tipo de estomia e par- } \\
\text { ticipação na consulta } \\
\text { de enfermagem de } \\
\text { estomaterapia. }\end{array}$ \\
\hline
\end{tabular}
terapia.

\begin{tabular}{|c|c|c|c|c|c|c|}
\hline Artigo 3 & $\begin{array}{c}\text { Percepção dos } \\
\text { pacientes com } \\
\text { estomia intestinal em } \\
\text { relação às mudanças } \\
\text { nutricionais e estilo } \\
\text { de vida. }\end{array}$ & $\begin{array}{l}\text { Clarissa M; Luana } \\
\text { B; Maria E; et al. }\end{array}$ & $\begin{array}{l}\text { Descrever as percep- } \\
\text { ções dos estomizados } \\
\text { intestinais sobre as } \\
\text { mudanças no estilo } \\
\text { de vida e aspectos } \\
\text { nutricionais. }\end{array}$ & $\begin{array}{l}\text { Estudo explora- } \\
\text { tório e descritivo, } \\
\text { com abordagem } \\
\text { qualitativa. }\end{array}$ & $\begin{array}{l}\text { Há a necessidade de } \\
\text { acompanhamento } \\
\text { nutricional e psico- } \\
\text { lógico aos estomiza- } \\
\text { dos. }\end{array}$ & 2019 \\
\hline Artigo 4 & $\begin{array}{l}\text { Tecnologia para } \\
\text { o autocuidado da } \\
\text { saúde sexual e repro- } \\
\text { dutiva de mulheres } \\
\text { estomizadas. }\end{array}$ & $\begin{array}{l}\text { Andressa F; Ana } \\
\text { K; Francisca M; } \\
\text { et al. }\end{array}$ & $\begin{array}{l}\text { Validar uma tecno- } \\
\text { logia do tipo cartilha } \\
\text { impressa para o au- } \\
\text { tocuidado na saúde } \\
\text { sexual e reprodutiva } \\
\text { de mulheres estomi- } \\
\text { zadas. }\end{array}$ & $\begin{array}{l}\text { Estudo metodoló- } \\
\text { gico. }\end{array}$ & $\begin{array}{l}\text { A tecnologia educati- } \\
\text { va em questão foi va- } \\
\text { lidada segundo con- } \\
\text { teúdo e aparência, } \\
\text { constituindo-se um } \\
\text { recurso adequado, } \\
\text { confiável e de fácil } \\
\text { compreensão. }\end{array}$ & 2016 \\
\hline Artigo 5 & $\begin{array}{l}\text { A visão dos enfer- } \\
\text { meiros sobre as } \\
\text { práticas educativas } \\
\text { direcionadas as pes- } \\
\text { soas estomizadas. }\end{array}$ & $\begin{array}{l}\text { Vanessa C; Norma } \\
\text { V; Carolina C; } \\
\text { et al. }\end{array}$ & $\begin{array}{l}\text { Analisar o ponto de } \\
\text { vista dos enfermeiros } \\
\text { sobre as ações educa- } \\
\text { tivas realizadas com } \\
\text { as pessoas estomiza- } \\
\text { das. }\end{array}$ & $\begin{array}{l}\text { Pesquisa qualitati- } \\
\text { va e exploratória, } \\
\text { interpretativa e crí- } \\
\text { tica. }\end{array}$ & $\begin{array}{l}\text { Os enfermeiros apre- } \\
\text { sentavam uma prá- } \\
\text { tica humanizada e } \\
\text { objetivavam tornar o } \\
\text { processo educativo } \\
\text { mais dinâmico. }\end{array}$ & 2017 \\
\hline Artigo 6 & $\begin{array}{l}\text { Qualidade de vida } \\
\text { de pessoas com } \\
\text { estomias intestinais } \\
\text { de eliminação. }\end{array}$ & $\begin{array}{l}\text { Cynthia R; Elaine } \\
\text { M; Maria H; et al. }\end{array}$ & $\begin{array}{l}\text { Objetivou-se avaliar } \\
\text { a qualidade de vida } \\
\text { de pessoas com es- } \\
\text { tomias intestinais de } \\
\text { eliminação. }\end{array}$ & $\begin{array}{l}\text { Estudo descritivo e } \\
\text { transversal. }\end{array}$ & $\begin{array}{l}\text { As estomias intesti- } \\
\text { nais de eliminação } \\
\text { interferem na quali- } \\
\text { dade de vida, princi- } \\
\text { palmente nos âmbi- } \\
\text { tos físico e social. }\end{array}$ & 2017 \\
\hline Artigo 7 & $\begin{array}{l}\text { Aspectos sociode- } \\
\text { mográficos e clínicos } \\
\text { de estomizados in- } \\
\text { testinais provisórios. }\end{array}$ & $\begin{array}{l}\text { Janderson } \mathrm{C} \text {; } \\
\text { Adriana P; } \\
\text { Katia J; et al. }\end{array}$ & $\begin{array}{l}\text { Caracterizar aspectos } \\
\text { sociodemográficos e } \\
\text { clínicos de estomiza- } \\
\text { dos intestinais provi- } \\
\text { sórios. }\end{array}$ & $\begin{array}{l}\text { Estudo transversal, } \\
\text { de natureza quan- } \\
\text { titativa. }\end{array}$ & $\begin{array}{l}\text { O conhecimento das } \\
\text { características da } \\
\text { população atendida } \\
\text { contribui para o pla- } \\
\text { nejamento da assis- } \\
\text { tência }\end{array}$ & 2017 \\
\hline Artigo 8 & $\begin{array}{l}\text { Cuidado integral à } \\
\text { pessoa estomizada } \\
\text { na Atenção Básica } \\
\text { - Conhecimento e } \\
\text { atuação do enfer- } \\
\text { meiro. }\end{array}$ & $\begin{array}{c}\text { Lidiaine N; Ana P; } \\
\text { Maria N. }\end{array}$ & $\begin{array}{l}\text { Apreciar o conhe- } \\
\text { cimento e a atuação } \\
\text { do enfermeiro no } \\
\text { cuidado à pessoa es- } \\
\text { tomizada na atenção } \\
\text { básica. }\end{array}$ & $\begin{array}{l}\text { Estudo com caráter } \\
\text { qualitativo, explo- } \\
\text { ratório e descriti- } \\
\text { vo. }\end{array}$ & $\begin{array}{l}\text { O ensino de enfer- } \\
\text { magem e a educação } \\
\text { permanente poderão } \\
\text { contribuir para uma } \\
\text { atuação competente e } \\
\text { eficaz de cuidado in- } \\
\text { tegral ao estomizado. }\end{array}$ & 2017 \\
\hline Artigo 9 & $\begin{array}{l}\text { Autoimagem e } \\
\text { autocuidado na } \\
\text { vivência de pacientes } \\
\text { estomizados: o olhar } \\
\text { da enfermagem. }\end{array}$ & $\begin{array}{c}\text { Daniela A; Rebeca } \\
\text { C; Naua R; et al. }\end{array}$ & $\begin{array}{l}\text { Analisar a percepção } \\
\text { de pacientes esto- } \\
\text { mizados sobre a sua } \\
\text { autoimagem e auto- } \\
\text { cuidado. }\end{array}$ & $\begin{array}{l}\text { Estudo explora- } \\
\text { tório, descritivo e } \\
\text { qualitativo. }\end{array}$ & $\begin{array}{l}\text { Recomenda-se que a } \\
\text { enfermagem se apro- } \\
\text { funde nos conheci- } \\
\text { mentos sobre esto- } \\
\text { mas, principalmente } \\
\text { em relação ao auto- } \\
\text { cuidado e aceitação } \\
\text { do paciente. }\end{array}$ & 2017 \\
\hline
\end{tabular}


Observa-se por meio da seleção dos artigos, como a confecção de uma colostomia afeta diretamente a vida diária dessas pacientes, como também a sexualidade, de forma que acaba por desencadear mudanças na rotina, alteração na fisiologia, autoestima, autoimagem, dificuldades no autocuidado, insegurança consigo mesmas e em relação aos parceiros. Após a cirurgia, pacientes demonstram-se muito abaladas emocionalmente e fisicamente, expressam-se menos atraentes, sensuais, afetando negativamente na vivência da sexualidade, reconhecendo-se também, incapazes de voltarem às suas rotinas de forma sucedida.

Após a análise dos artigos, emergiram as seguintes categorias temáticas: o viver da mulher colostomizada; as modificações na expressão da sexualidade; conhecimento para o autocuidado.

\section{$O$ viver da mulher colostomizada}

Um misto de sentimentos como medo e receios são experimentados pelo sujeito colostomizado, sentimentos esses que vão desde o desprezo da família e amigos, às dificuldades em lidar com a estomia, obstáculos para reintegração social e perda do emprego. Ameaça a sua integridade e situações de constrangimento geram um desequilíbrio emocional que interferem na aceitação de sua nova realidade de vida.

Em um estudo, evidenciou-se as modificações no viver da mulher estomizada, destacando as dificuldades que as mulheres participantes do estudo apresentaram para se adaptarem à estomia, principalmente, referente à eliminação de gases e privação do controle fecal. Após a cirurgia construtora de estoma, mudanças corporais e atividades cotidianas acometem o indivíduo, e cuidados específicos são necessários principalmente com a presença de um equipamento coletor para a estomia, levando a pessoa estomizada a vivenciar sentimentos negativos sobre seu corpo, induzindo a autoestima e os relacionamentos interpessoais. ${ }^{14,15}$

A necessidade de cuidados com a bolsa de colostomia, as modificações gastrintestinais, o aparecimento de sentimentos conflituosos, dificuldades e preocupações para lidar com a nova condição levaram os sujeitos estomizados investigados a visualizarem suas mudanças e limitações ocorridas no seu dia a dia. ${ }^{16}$ Dessa forma, além das complicações emocionais, uma cadeia de alterações de ordem física é gerada devido à estomia, fazendo com que o sujeito estomizado aprenda a conviver com as alterações corporais, como a cicatriz cirúrgica, nova maneira de evacuar ou urinar, presença de um estoma abdominal. ${ }^{17}$

Realizar o cuidado diário da estomia, da bolsa coletora e seus adjuvantes manifestou-se como uma tarefa difícil para as mulheres, pela necessidade de manusear diretamente suas eliminações, podendo experimentar sentimentos negativos ao processarem as transformações de seu corpo, sua vida, sua imagem, sendo a reabilitação um processo difícil e complexo. ${ }^{18}$

As mulheres passam por diversas modificações após a cirurgia construtora do estoma. Dentre essas, estão os hábitos alimentares que são ajustados para aumentar a consistência das fezes, reduzir o seu volume, minimizando também os odores das fezes e gases. Um estudo projetado em identificar a influência dos hábitos alimentares no controle das funções intestinais e as interferências no convívio social evidenciou a importância do conhecimento acerca do funcionamento do próprio corpo e a capacidade que essas desenvolvem para perceber a influência dos hábitos alimentares nas características e regulação de suas eliminações intestinais. ${ }^{19} \mathrm{Um}$ ponto positivo é que, após a estomia, para que problemas sejam evitados com o estoma, vários pacientes tornam-se mais criteriosos na escolha dos alimentos, incluindo na dieta alimentos mais saudáveis, realizando mastigação completa, refeições reduzidas em volume e mais frequentes, propiciando a manutenção ou recuperação do estado nutricional. ${ }^{20}$

Dessa forma, na tentativa de atrapalhar o funcionamento normal do intestino e para não ocorrer a presença de dejetos na bolsa coletora, muitos estomizados acabam realizando hábitos inadequados, como, realizarem poucas refeições e não consumirem outros grupos alimentares, gerando como consequência, transformações no estado nutricional e $\mathrm{o}$ mal funcionamento do organismo. ${ }^{20}$

Além das alterações na alimentação ocasionadas pela cirurgia construtora do estoma, os estomizados relatam mudanças cotidianas que requerem adaptação para serem experimentadas, como por exemplo, a prática de esportes, os afazeres domésticos, atividades de lazer, a sexualidade e o exercício do trabalho. Estudos realizados com estomizados indicaram que as mudanças cotidianas mais comumentes estão relacionadas à dificuldade de vivenciar a sexualidade, a atividades de lazer e ao retorno ao trabalho..$^{21,22}$

O trabalho tem papel fundamental na vida de qualquer indivíduo, e não o exercer poderá ser visto como perda de autonomia e independência pelo fato de, socialmente, apresentar uma carga moral e levar o indivíduo a sentir-se menosprezado e sujeito à discriminação. ${ }^{23}$ No entanto, o retorno ao trabalho faz com que os estomizados sintam-se ativos, possibilita o convívio com outras pessoas e contribui para a saúde mental e o bem-estar ${ }^{22,23}$, sendo assim, cabe aos profissionais das equipes que acompanham as pessoas com estomia conduzi-las e prepará-las para o retorno ao trabalho. ${ }^{22}$ Ressalta-se que logo após a adaptação do estomizado à sua nova situação de vida que fará com que ele se sinta confiante para retomar as atividades sociais e ao trabalho. ${ }^{22}$ 


\section{As modificações na expressão da sexualidade}

A mudança física que a presença do estoma ocasiona na mulher pode afetar a percepção que ela tem de si, prejudicando sua própria identidade, a forma como se enxergam podendo induzi-las ao isolamento social.

$\mathrm{O}$ isolamento social é frequentemente associado à estomia. A baixa satisfação com a vida e a ausência de apoio emocional e social estão conectadas ao isolamento. ${ }^{24} \mathrm{~A}$ mulher estomizada muitas vezes prefere se retirar do convívio social com amigos, colegas de trabalho, família, por conta da insegurança e receio de passar por situações constrangedoras, como por exemplo, o vazamento da bolsa coletora, o que necessita de cuidados a mais com esta e o estoma. No entanto, evidenciou-se em uma pesquisa que as mulheres com estomia declaram não se sentirem diferentes ou excluídas, mas que as pessoas de seu convívio se preocupam demais e perguntam quando a bolsa será retirada. ${ }^{25}$

Compreende-se ainda, que a presença da estomia afeta na expressão da sexualidade como um todo, até mesmo no desempenho sexual. A maior parte das pacientes colostomizadas apresenta algum tipo de dificuldade no contexto da sexualidade, seja relacionada aos distúrbios fisiológicos resultantes dos procedimentos cirúrgicos - como a redução ou perda da libido, dispareunia, ressecamento ou estenose vaginal ${ }^{10,12}$ - seja relacionada ao abalo psicológico decorrente da mudança da imagem corporal, evidenciado por sentimento de insegurança e vergonha do próprio corpo. ${ }^{26}$

Após a cirurgia de estomização, as percepções sobre a sexualidade são influenciadas pela orientação sexual dos envolvidos, a atitude eareação do companheiro e valores culturais destes. ${ }^{27}$ Tanto os homens como as mulheres enfrentam problemas com a intimidade e a sexualidade. ${ }^{28}$ Entretanto, as mulheres comparadas aos homens, demonstram maior preocupação com o corpo sexualmente valorizado e desejado pela sua estética do que por sua funcionalidade. ${ }^{29}$

Insegurança, eliminação involuntária de gases, o odor, medo de a bolsa estourar e o medo de rejeição, são as principais dificuldades que surgem principalmente quando se refere ao parceiro. ${ }^{30}$ Dessa forma, um estudo identificou que as mulheres estomizadas relataram mudanças na vida sexual, por preocupações com o parceiro, pois é frequente o sentimento de que a bolsa poderá estourar ou descolar. ${ }^{25}$

Estudos mostram que os estomizados demoram reassumir a atividade sexual tanto pelas complicações cirúrgicas como pela vergonha de sua nova imagem corporal, no entanto nota-se que a baixa autoestima se sobrepõe à limitação física. ${ }^{31,32}$ Tanto o indivíduo portador de estomia intestinal quando seu parceiro sexual necessita de ma1s informaçóes em relação a sua sexualidade. A inserção desse assunto, na rotina de atendimento da equipe multiprofissional e no planejamento de orientações, auxilia na melhora da qualidade de vida desses sujeitos. ${ }^{33}$

\section{Conhecimento para o autocuidado}

Essa categoria está associada às orientações que alguns pacientes recebem no pré e pós-operatório, descrevendo o conhecimento obtido a partir de cuidados prestados pelos profissionais de enfermagem e informações oferecidas por eles, ressaltando a importância da equipe de saúde no âmbito hospitalar diante ao processo de orientação para o autocuidado.

Ao promover um novo conhecimento após a colostomia, os sujeitos vivenciam o sentimento de se aceitar como um ser transformado, o que thes atribui um reparo de sua imagem e autoconceito, assim como o enfrentamento de diversas complicações que necessitam ser informadas pelo profissional de saúde para que o paciente possa desempenhar o autocuidado. ${ }^{34}$

É crucial uma educação em saúde para impedir complicações e agravos em relação ao manejo inadequado da colostomia. Portanto, a equipe de enfermagem representa uma conexão importante na educação em saúde e na capacitação do sujeito quanto ao autocuidado. ${ }^{35}$

O ensino do autocuidado é muito importante no processo de reabilitação do indivíduo com estomia, pois a pessoa torna-se participativa de seu cuidado e se adequa mais facilmente à nova condição, desenvolvendo atividades que visam prevenir agravos, promover sua qualidade de vida e recuperar a saúde. ${ }^{36,37}$

A pessoa com estomia requer orientações gerais sobre sua nova situação de vida, englobando os aspectos biopsicossociais, que variam desde as mudanças físicas centradas na redução do controle esfincteriano e dependência do equipamento coletor, até o estímulo à superação das dificuldades emocionais à procura da inclusão social. ${ }^{38,39}$

Aprender a acostumar-se com um estoma é um desafio e exige adaptação contínua às mudanças. Nos dias de hoje existem tecnologias de bolsa coletoras que colaboram para a melhoria da qualidade de vida. Uma destas tecnologias é o método de irrigação ${ }^{16}$ que auxilia os estomizados nas tarefas cotidianas. Esse método é utilizado para a normalização da atividade intestinal do sujeito que possui colostomia, é feito por meio da introdução de um volume de líquido no intestino grosso para expelir o conteúdo fecal em horário e dia planejado, e, dessa forma, fazendo com que os colostomizados se isentem do uso constante da bolsa coletora. ${ }^{40}$

Em um estudo transversal, realizado com intuito de comparar e avaliar a qualidade de vida 
de indivíduos com colostomia, que utilizam ou não o método de irrigação intestinal, evidenciou que as pessoas colostomizadas que praticam irrigação têm qualidade de vida consideravelmente melhor do que aquelas que não realizam. Entretanto, não são todas as pessoas estomizadas que estão aptas para realizar tal procedimento, pois, além do interesse e da motivação, a colostomia precisa ser terminal, de uma boca, situada no cólon descendente ou sigmoide, o paciente não deve apresentar complicação no estoma e não ser portador da síndrome de cólon irritável. ${ }^{19}$

Portanto, a implementação de tecnologias oclusoras e de irrigação, para indivíduos com colostomias descendentes e sigmoide, pode promover ampla escolha de gestão do autocuidado, aprimorando a imagem corporal, recuperando a continência por até 12 horas, possibilitando liberdade e amenizando o medo e o nervosismo provocado nas relações interpessoais. ${ }^{41}$

Devido ao conteúdo corrosivo que sai com as fezes, os estomas intestinais requerem uma atenção especial, causando a perda da integridade da pele. Dessa forma, o conhecimento sobre cuidados essenciais com os estomas remete-se à higienização periestomal, cuidado com os sinais flogísticos que podem surgir e com a pele íntegra. O cuidado com a pele periestomal deve ser um cuidado contínuo, devido a problemas como dermatites de contato e escoriações que ocorrerem com facilidade. $\mathrm{O}$ conhecimento do enfermeiro sobre as mudanças de pele traz ao usuário mais autonomia e confiança para o autocuidado, tornando-o mais otimista para desempenhar os cuidados em seu domicílio. ${ }^{42}$

A cor caracteriza a perfusão do estoma, uma tonalidade escura, acastanhada ou preta é um indicador de isquemia e necrose. O edema inicial é uma reação esperada em consequência do procedimento cirúrgico, mas desaparece após o $5^{\circ}$ ao $7^{\circ}$ dia de pós-operatório. Pequenas hemorragias também são presumidas, desde que sejam em menor quantidade. Além disto, em estomias intestinais não existem terminações nervosas, por esse motivo, as pessoas não sentem dor, logo cuidados devem ser tomados com traumas e na hora de realizar a higiene, por ser uma mucosa que pode ser lesionada com facilidade. ${ }^{43,44}$

A avaliação do domínio dos pacientes sobre sua capacidade de se auto cuidar e de desempenhar atividades de vida diária deve acontecer constantemente, pois é a partir dessa avaliação sistemática que o enfermeiro se baseia para implementar um plano de cuidados adaptado às reais necessidades dos indivíduos, além de estimar o nível de compreensão quanto às orientações fornecidas. ${ }^{45}$

Nas consultas de enfermagem, a presença contínua das pessoas com estomia contribui com a adaptação à existência do estoma, ainda, verifica-se a compreensão das modificações ocorridas em suas vidas, colabora-se com o processo de reabilitação e inclusão social. Ainda, constata-se que favorece o estreitamento da ligação entre educador e educando, beneficiando o processo educativo. 46

\section{Considerações finais}

Nota-se que o processo de estomização interfere agressivamente na vida de mulheres, modificando totalmente o estilo de vida e sua forma de expressar a sexualidade. Há, ainda, a dificuldade de aceitação do estoma, controle de fezes e gases, o processo de retorno ao trabalho, a realização de atividades do dia a dia e atividades de lazer, como também dificuldades apresentadas para o desempenho do autocuidado.

Ressalta-sea importância do apoio do parceiro e também de familiares, amigos e colegas de trabalho, para a reintegração no convívio social e restabelecimento das atividades diárias, de forma gradual, sempre respeitando seu tempo e espaço.

Quanto à sexualidade, percebe-se que as mulheres demonstram insegurança e vergonha por sua nova condição de saúde e medo da rejeição do parceiro, dificultando retomar a atividade sexual de maneira prazerosa, sendo a estomia considerada uma barreira na expressão da sexualidade. As mulheres necessitam de um tempo para aceitar a alteração corporal preparandose emocionalmente para reassumir a vida sexual.

Portanto mostra-se a importância do profissional enfermeiro estomaterapeuta no acompanhamento do paciente estomizado, proporcionando uma educação em saúde eficaz e instruções necessárias quanto ao autocuidado, assim fortalecendo sua autonomia, fazendo com que o indivíduo estomizado tenha cuidados necessários com a estomia e com a pele periestomal, proporcionando a recuperação da autoestima e colaborando para a reabilitação e inclusão social.

\section{Referências}

1. Rocha JJR. Estomas intestinais (ileostomias e colostomias) e anastomoses intestinais Med (Ribeirão Preto). 2011[citado em 2016 maio 16];44(1):51-6. Disponível em: http://revista.fmrp.usp.br/2011/vol44n1/ Simp5_Estomas intestinais.pdf.

2. Santana JCB, Souza AB, Dutra BS. Percepções de um grupo de enfermeiras sobre o processo do cuidar de pacientes portadores de ostomia definitiva. Rev. enferm. UFPE online. 2011; 5(7):1710-15. Disponível em: http://www.revista.ufpe.br/revistaenfermage m/index.php/revista/article/ viewArticle/1722.

3. Carvalho, SORM, Budó MLD, Silva MM, Alberti GF, Simon BS. "With some care, we can go on": Experiences of people with ostomy. Texto Contexto Enferm [Internet]. 2015 [acesso 2017 Dez 17];24(1):279-87. Disponível em: https://dx.doi.org/10.1590/0104-07072015003710013.

4. Silva CR, Andrade EM, Luz MH, Andrade JX, Silva GR. Quality of life of people with intestinal stomas. Acta Paul Enferm [Internet]. 2017 [acesso 2017 Dez 11];30(2):144-51. Disponível em: http://www.scielo.br/ pdf/ape/v30n2/en 1982-0194-ape-30-02-0144.pdf.

5. Mota MS, Gomes GC, Petuco VM. Repercussions in the living process of people with stomas. Texto Contexto Enferm [Internet]. 2016 [acesso 2017 Dez 18];25(1):e1260014. Disponível em: https://dx.doi.org/10.1590/0104- 


\section{4 .}

6. Pinto IMS. Desenvolvimento da competência de autocuidado da pessoa com ostomia de eliminação intestinal: validação do formulário [Dissertação]. Porto: Escola Superior de Enfermagem do Porto; 2014

7. Figueiredo PA, Alvim NAT. Guidelines for a Comprehensive Care Program to Ostomized Patients and Families: a Nursing proposal. Rev Latino-Am Enfermagem [Internet]. 2016 [acesso 2018 Jul 24];24:e2694. Disponível em: http://www.scielo.br/pdf/rlae/v24/0104-1169-rlae-24-02694. pdf.

8. Nugent KP, Daniels P, Stewart B, Patankar R, Johnson CD. Quality of life in stoma patients. Dis Colon Rectum [Internet]. 1999 Dec; [cited 2017 Aug 15]; 42(12):1569-74. Available from: https://www.ncbi.nlm. nih.gov/ pubmed/10613475.

9. Martins CG. Sexualidade da pessoa com ostomia de eliminação intestinal: que intervenção? [Dissertation]. Coimbra: Escola Superior de Enfermagem de Coimbra; 2013.

10. Silva AL, Monteiro PS, Sousa JB, Vianna AL, Oliveira PG. Partners of patients having a permanent colostomy should also receive attention from the healthcare team. Colorectal Disease [Internet]. 2014[cited 2015 Feb 12];16:O431O434. Available from: http://onlinelibrary.wiley.com/ doi/10.1111/codi.12737/epdf.

11. Mota MS, Gomes GC. Mudanças no Processo de Viver do Paciente Estomizado Após a Cirurgia. Revenferm UFPE online. 2013; 7(esp):707481. Disponível em: http://www.revista.ufpe.br/revistaenfermage $\mathrm{m} / \mathrm{index}$. $\mathrm{php} /$ revista/article/viewFile/3435/p df_4260.

12. Cardoso DBR et al. Sexualidade de pessoas com estomias intestinais. Rev Rene. 2015; 16(4):576-85. Disponível em: http://www.revistarene.ufc. br/revista/index. php/revista/article/viewFile/2051/pdf.

13. Kimura CA, Kamada I, Guilhem D, Fortes RC. Perception of sexual activities and the care process in ostomized women. Jornal ofColoproctology. 2013; 33(3):145150.Disponível em: http:/www.ncbi.nlm.nih.gov/pmc/ articles/P MC3573471/.

14. Marquis P, Marrel A, Jambon B. Quality of life in patients with stomas: the Montreux Study. OstomyWoundManage. 2003[citado em 2016 maio 16];49(2):48-55. Disponível em: http://www.owm.com/content/ qualitylifepatientswithstomasthemontreuxstudy.

15. Sales CA, Violin MR, Waidman MAP, Marcon SS, Silva MAP. Sentimentos de pessoas ostomizadas: compreensão existencial. RevEscEnferm USP. 2010[citado em 2016 maio 16];44(1):221-7. Disponível em: http://www. scielo.br/pdf/reeusp/v44n1/a31v44n1.pdf.

16. Cetolin SF, Beltrame V, Cetolin SK, Presta AA. Dinâmica sóciofamiliar com Pacientes Portadores de ostomia intestinal definitiva. ABCD arq. bras. cir. dig. 2013; 26(3):170-72. Disponível em: http://www.scielo.br/ pdf/abcd/v26n3/03.pdf

17. Mota M, Gomes G, Silva CD, Gomes VL, Pelzer M, Barros E. Autocuidado: uma estratégia para a qualidade de vida da pessoa com estomia. InvestigEnferm. ImagenDesarr. 2016;18(1): 63-78. Disponível em: http://dx.doi.org/10.11144/Javeriana.ie181.aeqv.

18. Mattos M, Maruyama SAT. A experiência de uma pessoa com doença renal crônica em hemodiálise. Rev. gaúcha enferm. (Online). 2010; 31(3):428-34. Disponível em: http://www.scielo.br/pdf/rgenf/v31n3/v31n3 a04.pdf.

19. Cesaretti IUR, Santos VLCG, Vianna LAC. al. Qualidade de vida de pessoas colostomizadas com e sem uso de métodos de controle intestinal. RevBrasEnferm, Brasília 2010 jan-fev; 63(1): 16-21. Disponível em http://www.scielo.br/scielo.php?script=sci_ar ttext\&pid=S003471672010000100003 .

20. Silva DG, Bezerra ALQ, Siqueira KM, Paranaguá, TTB, Barbosa MA. Influência dos hábitos alimentares na reinserção social de um grupo de estomizados. RevEletrEnferm [Internet]. 2010 [acesso 2017 Dez 15];12(1):56-62. Disponível em: https://www.fen.ufg.br/revista/v12/n1/ v12n1a07.htm.

21. Nascimento CMS, Trindade GLB, Luz MHBA, Santiago RF. Vivência do paciente estomizado: uma contribuição para a assistência de enfermagem. Texto Contexto Enferm [Internet]. 2011 [acesso 2017 Dez 28];20(3):557-64. Disponível em: https://dx.doi.org/10.1590/S0104-07072011000300018.
22. Mauricio VC, Souza NVDO, Lisboa MTL. The nurse in the rehabilitation of the person with a stoma. Esc Anna Nery [Internet]. 2013 [acesso 2017 Dez 28];17(3):416-22. Disponível em: http:// www.scielo.br/ pdf/ean/v17n3/en_1414-8145-ean-17-03-0416.pdf.

23. Souza PCM, Costa VRM, Maruyama SAT, Costa ALR, Rodrigues AEC, Navarro JP. As repercussões de viver com uma colostomia temporária nos corpos: individual, social e político. Rev. EletrEnf [Internet]. 2011 [acesso 2017 Dez 28];13(1):50-9. Disponível em: http://www.fen.ufg.br/ revista/ v13/n1/v13n1a06.htm.

24. Nichol S. Social connectivity in those 24 months or less postsurgery. J WoundOstomyContinenceNurs. 2011; 38(1):63-8. Disponível em: http:// www.ncbi.nlm.nih.gov/pubmed/21287 772 .

25. Moraes JT, Sousa LA, Carmo WJ. Análise do autocuidado das pessoas estomizadas em um município do centrooeste de minas gerais. RevenfermCent.-Oeste Min. 2012; 2(3):337346. Disponível em: http://www. seer.ufsj.edu.br/index.php/reco m/article/viewArticle/224.

26. Cerezett CRN. Psychological Instructions and reactive capacity of ostomized individuals and their relatives. Mundo Saúde [Internet]. 2012[cited 2013 Mar 8];36(2):332-9. Availablefrom:http://bvsms.saude.gov. br/bvs/artigos/mundo saude/orientacoes piscologicas capacidade reativa pe ssoas.pdf

27. Li CC. Sexuality among patients with a colostomy: an exploration of the influences of gender, sexual orientation, and asian heritage. J Wound Ostomy Continence Nurs. 2009; 36(3):299-305. Disponívelem: http://www. ncbi.nlm.nih.gov/pubmed/19448 510 .

28. Grant M, McMullen CK, Altschuler A, Mohler MJ, Hornbrook MC, Herrinton LJ, Wendel CS, et al. Gender differences in quality of life among long-term colorectal cancer survivors with ostomies. Oncol Nurs Forum. 2011; 38(5):587-96. Disponível em:http://www.ncbi.nlm.nih.gov/pmc articles/P MC3251903/.

29. Abbott BD, Barber BL. Embodied image: gender differences in functional and aesthetic body image among Australian adolescents. BodyImage. 2010; 7(1):22-31. Disponível em: http://www.sciencedirect. com/science/articl e/pii/S1740144509001053.

30. Cassero PAS, Aguiar JE. Percepções emocionais influenciadas por uma estomia. Revista Saúde e Pesquisa. 2009; 2(2):23-7. Disponível em: http://periodicos.unicesumar.edu.br/index.p hp/saudpesq/article/ viewFile/1058/780

31. Calcagno GG, Peres BP, Pizarro ADR, Pereira MA, Silva CE, Gomes OVL. Ser mujerconostomía: lapercepción de lasexualidad. Enferm Global [Internet]. 2012 [acesso 2017 Dez 29];11(27):2233. Disponível em: http:// scielo.isciii.es/pdf/eg/v11n27/clinica2.pdf.

32. Vera SO, Sousa GN, Araújo SNM, Moreira WC, Damasceno CKC, Andrade EML. Sexuality of patients with bowel elimination ostomy. Rev Fund Care Online [Internet]. 2017 [acesso 2017 Dez 29];9(2):495-502. Disponível em: http://www.seer.unirio.br/index.php/cuidadofundamental/ article/view/5451/pdf.

33. Braga RCD, Almeida CE, Santana ME, Seabra CD, MSH, Okino SN Sexualidade de pessoas com estomias intestinais. Rev Rene [Internet]. 2015 [acesso 2017 Jul 23];16(4):576-85. Disponível em: http://www.redalyc.org/ pdf/3240/324041519015.pdf.

34. Umpiérrez AF, Fort ZF. Family of experiences of patients with colostomy and expectations of professional intervention. Rev LatinoAmEnferm. 2014[citado em 2016 jul. 11];22(2):241-7. Disponível em: http://www.scielo. br/scielo.php?script=sci arttext\&pid=S010411692014000200241.

35. Moraes AA, Balbino CM, Souza MMT. O desconforto em pacientes ostomizados. Revista Pró-UniverSUS. 2015[citado em 2016 set. 20];6(1):58. Disponível em: http://www.uss.br/pages/revistas/revistaprouniversus/ V6N12015/pdf/001.pdf.

36. Faria MGA, David HMSL, Acioli S. A educação a distância no Brasil e a sua influência na saúde e na enfermagem: aspectos históricos. CadCiênc Saúde. 2014;3(1):87-93.

37. Menezes LCG, Guedes MVC, Oliveira RM, Oliveira SKP, Meneses LST, Castro ME. Prática de Autocuidado de estomizados: contribuições da Teoria de Orem. Rev Rene [Internet]. 2013; [cited 2015 Jun 26]; 14(2):30110. Available from: http://www.revistarene.ufc.br/revista/ index.php/revista/ 
$\operatorname{article/view/235.~}$

38. Cheng F, Meng AF, Yang LF, Zhang YN. The correlation between ostomy knowledge and self-care ability with psychosocial adjustment in Chinese patients with a permanent colostomy: a descriptive study. Ostomy Wound Manage [Internet]. 2013 Jul; [cited 2015 Aug 2]; 59(7):35-8. Available from: https://www.ncbi.nlm.nih.gov/pubmed/23846005.

39. Lopes APAT, Decesaro MN. Adjustments experience by persons with an ostomy: a integrative review of the literature. Ostomy Wound Manage [Internet]. 2014 Oct; [cited 2017 Aug 31]; 60(10):34-42. Available from: https://www.ncbi.nlm.nih.gov/pubmed/?term=Adjustments+experienc e+by + persons + with + an + ostomy $\% 3 \mathrm{~A}+\mathrm{a}+$ integrative + review + of + the +1 iterature.

40. Karadağ A, Menteş BB, Ayaz S. Colostomy irrigation: results of 25 cases with particular reference to quality of life. J ClinNurs [Internet]. 2005 [acesso 2017 Dez 30];14(4):479-85. Disponível em: https://www.ncbi.nlm. nih.gov/pubmed/15807755.

41. Swan E. Colostomy, management and quality of life for the patient. Br J Nurs. 2011; 20(1):22, 24-8.

42. Martins PAF, Alvim NAT. Perspectiva educativa do cuidado de enfermagem sobre a manutenção da estomia de eliminação. Rev Bras Enferm. 2011; 64(2):322-7.

43. Golicki D, Styczen P, Szczepkowski M. Quality of life in stomapatients in Poland: multicenter cross-sectionalstudyusing WHOQOLBREF questionnaire. PrzeglEpidemiol. 2013; 67(3):491-6.

44. Burch J. Resuming a normal life: holistic care of the person with an ostomy. Brit J Community Nurs. 2011; 16(8):366-73.

45. Coelho AR, Santos FS, Poggetto MTD. Stomas changing lives: Facing the illness to survive. Rev Min Enferm [Internet]. $2013 \mathrm{Apr} / \mathrm{Jun}$; [cited 2017 Jul 20]; 17(2):268-77. Available from: http://www.reme.org.br/ artigo/detalhes/649. DOI: 10.5935/1415-2762.20130021.

46. Grant M, McCorkle R, Hornbrook MC, Wendel CS, Krouse R. Development of a chronic care ostomy self-management program. J Cancer Educ [Internet]. 2013 Mar; [cited 2015 Jun 26]; 28(1):708. Available from: https:/www.ncbi.nlm.nih.gov/pmc/articles/ PMC3578127/. DOI: 10.1007/ s13187-012-0433-1. 